\title{
Assessment of the effect of psoriasis on male sexual function
}

\section{Original Article}

\author{
Mahmoud Y. Abd El-Mawla', Abdalla Kandil', Ahmed A. Hashim ${ }^{1}$,Al-Sayed Zidan ${ }^{2}$ \\ ${ }^{1}$ Departments of Dermatology, Venereology and Andrology \\ ${ }^{2}$ Diagnostic Radiology, Faculty of Medicine, Zagazig University, Zagazig, Egypt
}

\begin{abstract}
Background: Only $9 \%$ of the patients with psoriasis are satisfied with sexual function, whereas $43 \%$ perceive it as insufficient. The etiology of erectile dysfunction (ED) in male patients with psoriasis is likely to be multifactorial. The psychological problems associated with psoriasis highly contribute to ED in patients with psoriasis. Moreover, an increased prevalence of the core components of the metabolic syndrome, including obesity, dyslipidemia, and glucose intolerance, contributes to the ED seen in patients with psoriasis.

Aim: To clarify the effect of psoriasis on male sexual function.

Patients and methods: This study was conducted on 62 married male individuals aged between 20 and 40 years. They were allocated into two equal groups: 31 male patients with psoriasis in group I, and 31 male apparently healthy controls in group II. All patients were evaluated by the International Index of Erectile Function (IIEF-5). However, only 10 patients with psoriasis accepted to undergo penile duplex ultrasonography, and it was conducted in 10 healthy controls as well.

Results: There was a highly significant increase in the prevalence of ED in patients with psoriasis over the control group. There was a highly significant decrease in the values of the IIEF-5 among patients with psoriasis, which was less than the values of the control group. Moreover, there was a highly significant correlation between psoriasis area and severity index scores with the IIEF-5 scores and a significant difference in the results of penile duplex ultrasound among the two studied groups.

Conclusion: Male patients with psoriasis have a higher prevalence of ED associated with increased risk of sexual dysfunction in those patients.
\end{abstract}

Key Words: Impotence, male sexual dysfunction, psoriasis

Received: 27 September 2018, Accepted: 10 November 2018

Corresponding Author: Abdalla Kandil, MD, Department of Dermatology, Venereology and Andrology, Faculty of Medicine, Zagazig University, Zagazig, Egypt, Tel.: +20 120511 5291, E-mail: ak.kandil@hotmail.com

ISSN: 2090-6048, June 2018, Vol. 8, No. 2

\section{INTRODUCTION}

Psoriasis is considered as a persistent inflammatory skin disorder, and it is well known that psoriasis can have a high negative effect on patients'quality of life, with physical, social, and psychological effect that can be compared with that found in cardiovascular disease, diabetes mellitus, and malignancy ${ }^{[1]}$.

Patients with psoriasis have an increased risk of cardiovascular comorbidities compared with a control population $^{[2]}$.

Many studies found increased rates of coronary heart disease in patients with psoriasis. There is a significantly increased risk of major cardiac events in patients with severe psoriasis ${ }^{[3]}$.

There is increasing evidence that psoriasis is associated with metabolic syndrome ${ }^{[4]}$. It was reported that there is a significant increased risk of metabolic syndrome in patients with psoriasis compared with controls even after adjustment for age, sex, race/ ethnicity, smoking, and C-reactive protein levels ${ }^{[5]}$.
Genetic susceptibility and overlapping inflammatory pathways may be potential biological links underlying this association. The chronic and systemic T-helper-1-mediated and T-helper-17mediated inflammation of psoriasis characterized by increased levels of proinflammatory cytokines, such as tumor necrosis factor- $\alpha$ and interleukin- 6 , not only promotes epidermal hyperplasia in psoriasis but also antagonizes insulin signaling, alters adipokine expression, and mediates insulin resistance and obesity ${ }^{[6]}$.

Only few studies looking at sexual function in patients with psoriasis were found; all of them reported an increased prevalence of sexual dysfunction in patients with psoriasis ${ }^{[7]}$. An Italian study of more than 900 patients found prevalence rates of sexual life impairment of between 35.5 and $71.3 \%$, depending on the questionnaire used ${ }^{[8]}$.

Erectile dysfunction (ED) has been defined as the persistent inability to attain and/or maintain an 
erection sufficient for sexual performance ${ }^{[9]}$. ED is very common, and its prevalence as well as severity increases with age ${ }^{[10]}$.

It is known that a main cause ofED is atherosclerosis affecting the pelvic blood vessels. The occurrence of $\mathrm{ED}$ is recognized to expect upcoming cardiovascular disease, and early diagnosis may allow appropriate alteration of modifiable risk factors, or help the detection of undiagnosed cardiovascular disease. There is an evolving link between psoriasis, metabolic syndrome, atherosclerotic disease, elevated rates of myocardial infarction and stroke seen in patients with psoriasis $^{[11]}$

The International Index of Erectile Function (IIEF) is a widely used, multidimensional self-report index for the evaluation of male sexual function. It has been recommended for diagnostic evaluation of ED severity ${ }^{[12]}$.

A modified five-item IIEF was developed (IIEF-5) to diagnose the presence and severity of ED. The five items selected were based on the ability to identify the presence or absence of ED and on adherence to the National Institute of Health's definition of ED. These items focused on erectile function and intercourse satisfaction $^{[13]}$.

AIM

This study aimed to clarify the effect of psoriasis on male sexual function, namely, erectile function.

\section{PATIENTS AND METHODS}

This study was conducted in Department of Dermatology and Venereology and Andrology, Faculty of Medicine, Zagazig University Hospitals, during the period from November 2015 to November 2016.

Ethical approval for this study (Institutional Review Board no. 2385) was provided by the Institutional Review Board for Faculty of Medicine, Zagazig University, Zagazig, on 22 October 2015.

Written informed consent was taken from the patients before the study.

Sixty-two male individuals were included in this study. Participants were allocated into two groups: group I had 31 patients with psoriasis and group II had 31 apparently healthy controls.

Inclusion criteria were married male patients with psoriasis (nonsmokers and nonalcohol abusers) and normal healthy control patients with age group $20-40$ years.

\section{Exclusion criteria}

Patients with penile abnormalities that could significantly impair erectile function such as significant curvature or severe hypospadias; chronic diseases such as pulmonary, hepatic, renal, hematological, neurologic, psychosis or similar psychiatric disorders and malignancy; and patients using antidepressants, diuretics, $\beta$-blockers, inhibitors of $5-\alpha$ reductase or other drugs that are known to interfere with erectile function were the exclusion criteria.

\section{Methods}

(1) Detailed history included personal history, present history, past history and family history; full general examination that includes BMI calculation, blood pressure measurement, and detection of associated systemic disorders; local examination of the penis and the scrotum, and dermatological examination that includes detection of the type of psoriasis and psoriasis area and severity index (PASI) score were performed. IIEF-5 was applied to all cases. Penile duplex ultrasonography and laboratory investigations including random blood sugar and lipid profile were applied to all patient.

\section{RESULTS}

There is no significant difference between age and BMI in both groups (Table 1), nonsignificant values between random blood sugar of both studied groups, and highly significant values between serum cholesterol, serum triglycerides, high-density lipoprotein-cholesterol, and low-density lipoproteincholesterol levels of both studied groups (Table 2).

A highly significant increase in the prevalence of ED in patients with psoriasis versus the control group was found (Table 3 ).

There was a highly significant difference between the values of the IIEF-5 in the two studied groups (Table 4).

Age was a nonsignificant cardiovascular risk factor for ED in patients with psoriasis, whereas BMI, random blood sugar, and lipid profile were significant risk factors (Table 5).

(Table 6) showed significant results regarding the penile duplex ultrasound results among the studied groups. 
Abd El-Mawla et al

Table 1: Demographic data of the studied groups

\begin{tabular}{lccccc}
\hline & Patients with psoriasis & Controls & t value & P value & Significance \\
\hline Age (years) & & & & & \\
$\quad$ Mean \pm SD & $35.3 \pm 4.5$ & $33.9 \pm 3.9$ & 1.36 & -17 & NS \\
$\quad$ Range & $26-40$ & $26-40$ & - & - & - \\
BMI & & & & & NS \\
Mean \pm SD & $30.1 \pm 6.4$ & $27.9 \pm 3.2$ & 1.72 & - & - \\
Range & $2.7-44.1$ & $23.2-37.4$ & - & - & - \\
Duration of psoriasis (years) & & - & - & - & - \\
Mean \pm SD & $7.7 \pm 7.5$ & - & - & - & - \\
Range & $0.5-28$ & - & - & - \\
Median & 4 & - & - & - \\
\hline
\end{tabular}

$P>0.05$, significant.

Table 2: Random blood sugar and lipid profile among studied groups

\begin{tabular}{|c|c|c|c|c|c|}
\hline & Patients with psoriasis & Controls & $\mathrm{t}$ value & $P$ value & Significance \\
\hline \multicolumn{6}{|c|}{ Random blood sugar } \\
\hline Mean \pm SD & $99.9 \pm 10.6$ & $96.6 \pm 7.4$ & 1.42 & 0.16 & NS \\
\hline Range & $97.6-121.4$ & $81.7-113.1$ & & & \\
\hline \multicolumn{6}{|c|}{ Serum cholesterol } \\
\hline Mean \pm SD & $207.7 \pm 36.6$ & $118.6 \pm 37.3$ & 4.7 & $>0.001$ & HS \\
\hline Range & $159.3-286.4$ & $143.3-211$ & & & \\
\hline \multicolumn{6}{|c|}{ Serum triglycerides } \\
\hline Mean \pm SD & $162.6 \pm 43.5$ & $118.6 \pm 37.3$ & 4.27 & $>0.001$ & HS \\
\hline Range & $76.6-241.1$ & $69.4-196.4$ & & & \\
\hline \multicolumn{6}{|c|}{ HDL-cholesterol } \\
\hline Mean \pm SD & $40.8 \pm 12.1$ & $61.4 \pm 10.5$ & 7.14 & $>0.001$ & HS \\
\hline Range & $19.6-70.1$ & $39.8-79.1$ & & & \\
\hline \multicolumn{6}{|l|}{ LDL-cholesterol } \\
\hline Mean \pm SD & $115.5 \pm 28.0$ & $73.7 \pm 13.2$ & 7.54 & $>0.001$ & HS \\
\hline Range & $65.2-185.1$ & $43.2-101.3$ & & & \\
\hline
\end{tabular}

HDL, high-density lipoprotein; HS, highly significant; LDL, low-density lipoprotein.

$P<0.05$, significant.

Table 3: Prevalence of erectile dysfunction among studied groups

\begin{tabular}{lccccc}
\hline IIEF-5 & $\begin{array}{c}\text { Patients with } \\
\text { psoriasis [n (\%)] }\end{array}$ & Controls [n (\%)] & $\chi^{2}$ value & P value & Significance \\
\hline ED $\leq 21$ & $18(58.1)$ & $2(6.5)$ & 18.9 & $>0.001$ & HS \\
Normal 21 & $13(41.9)$ & $29(93.5)$ & & \\
\hline
\end{tabular}

ED, erectile dysfunction; HS, highly significant; IIEF-5, International Index of Erectile Function-5.

$P<0.05$, significant.

Table 4: International Index of Erectile Function-5 score among studied groups

\begin{tabular}{lcccc}
\hline IIEF-5 score & Patients with psoriasis & Controls & t value & $P$ value \\
\hline Mean \pm SD & $19.7 \pm 3.76$ & $23.2 \pm 1.1$ & 4.9 & $<0.001$ \\
Range & $11-25$ & $21-25$ & & HS \\
\hline
\end{tabular}

HS, highly significant; IIEF-5, International Index of Erectile Function-5.

$P<0.05$, significant. 
Table 5: Cardiovascular risk factors for erectile dysfunction in patients with psoriasis

\begin{tabular}{|c|c|c|c|c|c|}
\hline Risk factors & Patients with ED $(n=18)[n(\%)]$ & Patients without ED $(n=13)[n(\%)]$ & OR $(95 \% \mathrm{CI})$ & $P$ value & Significance \\
\hline \multicolumn{6}{|l|}{ Age (years) } \\
\hline$<30$ & $3(16.7)$ & $3(23.1)$ & $1.5(0.19-13.31)$ & 0.9 & NS \\
\hline$>30$ & $15(83.3)$ & $10(76.9)$ & & & \\
\hline \multicolumn{6}{|l|}{ BMI } \\
\hline Average (18.5-29.9) & $2(11.1)$ & $7(53.8)$ & $9.33(1.19-91.8)$ & 0.003 & Significant \\
\hline Obese $(\geq 30)$ & $16(88.9)$ & $6(45.2)$ & & & \\
\hline \multicolumn{6}{|c|}{ Random blood sugar $[\mathrm{n}(\%)](\mathrm{mg} / \mathrm{dl})$} \\
\hline$>90$ & $7(38.9)$ & $10(76.9)$ & $5.24(0.85-36.38)$ & 0.03 & Significant \\
\hline$\geq 90$ & $11(61.1)$ & $3(23.1)$ & & & \\
\hline \multicolumn{6}{|l|}{ Lipid profile $[\mathrm{n}(\%)]$} \\
\hline Normal & $5(27.8)$ & $9(69.2)$ & $5.85(0.97-39.46)$ & 0.02 & Significant \\
\hline Hyperlipidemia & $13(72.2)$ & $4(30.8)$ & & & \\
\hline
\end{tabular}

CI, confidence interval; ED, erectile dysfunction; OR, odds ratio.

$P>0.05$, significant.

Table 6: Penile duplex ultrasound among studied groups

\begin{tabular}{lcccc}
\hline Penile duplex & $\begin{array}{c}\text { Patients with psoriasis } \\
(\mathrm{n}=10)[\mathrm{n}(\%)]\end{array}$ & $\begin{array}{c}\text { Controls }(\mathrm{n}=10) \\
{[\mathrm{n}(\%)]}\end{array}$ & $\chi^{2}$ value & Significance \\
\hline Normal & $3(30)$ & $10(100)$ & 10.77 & 0.004 \\
Arterial insufficiency & $6(60)$ & 0 & & Significant \\
Venous leakage & $1(10)$ & 0 & & \\
\hline
\end{tabular}

\section{DISCUSSION}

Psoriasis is a chronic inflammatory skin disease. It is increasingly recognized as a multisystem inflammatory condition associated with a range of co-morbid diseases and risk factors, including obesity, metabolic syndrome, cardiovascular disease, psoriatic arthritis, autoimmune disease, psychiatric illness, liver disease, smoking, alcohol abuse, and sexual dysfunction $^{[14]}$.

In the present study, 62 patients were included. They were divided into two equal groups: 31 patients with psoriasis in group I and 31 healthy controls in group II.

Only 10 patients with psoriasis accepted to undergo penile duplex ultrasonography. The results showed arterial insufficiency in six patients with psoriasis supporting the endothelial defect in patients with psoriasis.

In this study, there was a nonsignificant difference between age and BMI in both groups, but there was a highly significant increase in the prevalence of ED in patients with psoriasis over the control group.

The present study showed a highly significant decrease in the values of the IIEF-5 among patients with psoriasis, which were less than the values of the control group.
The present study showed that age was a nonsignificant cardiovascular risk factor for ED in patients with psoriasis, whereas BMI, random blood sugar, and lipid profile were significant risk factors.

Likewise, there was a highly significant correlation between PASI scores with the IIEF-5 scores, which reflects an association between the severity of psoriasis and increased risk of sexual dysfunction.

Similarly, our study showed a significant difference in results of penile duplex ultrasound among the two studied groups. Overall, three patients with psoriasis accepted to undergo penile duplex ultrasound: three $(30 \%)$ of them showed normal penile duplex, six $(60 \%)$ patients showed arterial insufficiency, and one $(10 \%)$ patient showed venous leakage. The number of healthy controls who accepted to undergo penile duplex ultrasound was 10 , and they all showed normal results $(100 \%)$.

Our results agreed with those reported by Abd El-Salam et al..$^{[15]}$ who evaluated 15 patients with psoriasis and 18 controls. They found that among the patients with psoriasis, only two $(13.3 \%)$ patients had normal erectile function, whereas $13(86.7 \%)$ patients with psoriasis had ED. Among them, one patient had mild ED, one had mild-moderate ED, five patients had moderate ED, and eight patients had severe ED. However, in the control group (18 patients), eight $(44.4 \%)$ patients showed normal erectile function and 


\section{$10(55.6 \%)$ patients showed ED $(\mathrm{P}=0.01)$.}

Moreover, the results of this study agreed with the results of Sampogna et al. ${ }^{[8]}$ in which their results showed that sexual impairment was very frequent in patients with psoriasis; IIEF total scores were found to be significantly decreased in male patients compared with healthy controls $(\mathrm{P}=0.023)$, in addition the more severe disease was associated with more sexual impairment $(\mathrm{P}=0.05)$.

Similarly, our study results were supported by the results approved by Tasliyurt et al..$^{[4]}$. They have observed 37 male patients with psoriasis and 28 healthy men. Metabolic syndrome was observed in $14(35.15 \%)$ patients and in four $(14.28 \%)$ controls. The frequency of metabolic syndrome was higher in patients with psoriasis compared with healthy controls, and the difference was statistically significant $(\mathrm{P}=0.032)$. IIEF score in patients with psoriasis $(17.76 \pm 6.61)$ was significantly lower than in control group $(23.46 \pm 4.71)(\mathrm{P}=0.005)$. ED was observed in $30(81.08 \%)$ patients in patients with psoriasis and in $15(53.57 \%)$ patients in control group. The difference was significant $(\mathrm{P}=0.018)$. IIEF score had significant negative correlations with PASI score.

Moreover, our study results are matched with results released by Ji et al. ${ }^{[16]}$ who found that the proportion of ED in patients with plaque psoriasis was significantly higher than in the control group (52.9 vs. $40.3 \%$, respectively; $\mathrm{P}=0.018$ ). ED was classified as mild in $19.9 \%$, mild to moderate in $17.3 \%$, moderate in $9.9 \%$, and severe in $5.8 \%$ of patients with plaque psoriasis, and as mild in $21.5 \%$, mild to moderate in $13.1 \%$, moderate in $4.2 \%$, and severe in $1.6 \%$ of control group participants. Differences in moderate $(\mathrm{P}=0.04)$ and severe $\mathrm{ED}(\mathrm{P}=0.053)$ were statistically significant between patients with plaque psoriasis and the control group.

Pietrzak et al. ${ }^{[17]}$ determined that the lipid disturbances are recognized as a very important part in the pathogenesis of psoriasis. The results of most studies are coherent and indicate that the increased total cholesterol, low-density lipoprotein-cholesterol, and triglycerides, decreased high-density lipoproteincholesterol in the serum of patients with psoriasis, and increased production of oxygen metabolites are features of the metabolic syndrome.

Psychiatric comorbidity is estimated to affect more than $30 \%$ of patients with dermatologic disease. The prevalence of depression and anxiety in those with psoriasis, specifically, is significantly higher than that observed in the general population, and in comparison, to many other dermatologic conditions, psychiatric morbidity is higher and quality of life scores are lower ${ }^{[18]}$.

Patients with psoriasis have a reduction in their quality of life similar to or worse than patients with other chronic diseases, such as ischemic heart disease and diabetes ${ }^{[19]}$.

It is also known that the psychological problems associated with psoriasis are highly likely to contribute to ED in patients with psoriasis ${ }^{[20]}$.

ED is itself a cardiovascular risk factor and is significantly related to cardiovascular factors such as hypertension, hyperlipidemia, diabetes mellitus, and atherosclerotic disease in patients with psoriasis. This means that we must pay attention to patients with psoriasis with cardiovascular risk factors, especially hypertension and hyperlipidemia ${ }^{[11]}$.

Psoriasis and atherosclerosis may have certain common underlying pathogenic mechanisms; although psoriasis has long been established as an inflammatory disease, the inflammatory nature of atherosclerosis has been highlighted later, and both are associated with $\mathrm{T}$ lymphocyte-mediated adaptive immune events, and mechanisms involving innate immunity ${ }^{[21]}$.

Specifically, both psoriasis and atherosclerosis are associated with T-helper-1 polarization of the adaptive cellular immune response, characterized particularly by $\mathrm{T}$-cell production of interferon-c in lesions in vivo. Moreover, the proinflammatory effects of T-helper-17 cells and suppressive effects of $\mathrm{T}$ regulatory subsets have also been implicated in both pathologies ${ }^{[22]}$.

To sum up, endothelial dysfunction in psoriasis explains the ED and metabolic disorders with psychogenic profile in patients with psoriasis could affect male sexual function.

\section{CONCLUSION}

Male patients with psoriasis have a higher prevalence of ED with increased risk of sexual dysfunction in those patients.

\section{ACKNOWLEDGEMENTS}

Authors thanks for Dr Monira Wakid, El Houd El Marsod Hospital for her support and effort.

\section{CONFLICTS OF INTEREST}

There are no conflicts of interest. 


\section{REFERENCES}

1. Gelfand JM, Weinstein R, Porter SB, Neiman AK, Berlin JA, Marqolis DJ. Prevalence and treatment of psoriasis in the United Kingdom: a population-based study. Arch Dermatol 2005; 141:1537-1541.

2. Pietrzak A, Bartosinska J, Chodorowska G, Schwartz R. Cardiovascular aspects of psoriasis: an updated review. Int $\mathrm{J}$ Dermatol $2013 ; 52: 153-162$.

3. Mehta NN, Yu Y, Pinnelas R, Krishnamoorthy $\mathrm{P}$, Shin DB, Troxel AB. Attributable risk estimate of severe psoriasis on major cardiovascular events. Am J Med 2011; 124:775.

4. Tasliyurt T, Bilir Y, Sahin S, Seckin HY, Kaya SU, Sivgin H. Erectile dysfunction in patients with psoriasis: potential impact of the metabolic syndrome. Eur Rev Med Pharmacol Sci $2014 ; 18: 581-586$.

5. Love TJ, Qureshi AA, Karlson EW, Gelfand JM, Choi HK. Prevalence of the metabolic syndrome in psoriasis: results from the National Health and Nutrition Examination Survey, 2003-2006. Arch Dermatol 2011; 147:419-424.

6. Davidovici BB, Sattar N, Prinz J, Puig L, Emery P, Barker JN, van de Kerkhof P. Psoriasis and systemic inflammatory diseases: potential mechanistic links between skin disease and co-morbid conditions. J Invest Dermatol 2010; 130:1785-1796.

7. Mercan S, Altunay IK, Demir B, Akpinar A, Kayaoglu S. Sexual dysfunctions in patients with neurodermatitis and psoriasis. J Sex Marital Ther 2008; 34:160-168.

8. Sampogna F, Gisondi P, Tabolli S, Abeni D. Impairment of sexual life in patients with psoriasis. Dermatology 2007; 214:144-150.

9. Hackett G, Kirby M, Wylie K, Heald A, OsseiGerning N, Edwards D, Muneer A. British Society for Sexual Medicine guidelines on the management of erectile dysfunction. J Sex Med 2008; 5:1841-1865.

10. Feldman HA, Goldstein I, Hatzichristou DG, Krane RJ, McKinlay JB. Impotence and its medical and psychosocial correlates: results of the Massachusetts Male Aging Study. J Urol 1994; 151:54-61.

11. Gandaglia G, Briganti A, Jackson G, Kloner RA, Montorsi F, Montorsi P. A systematic review of the association between erectile dysfunction and cardiovascular disease. Eur Urol 2014; 65:968-978.

12. Rosen RC, Cappelleri JC, Gendrano N. The International Index of Erectile Function (IIEF): a state-of-the-science review. Int $\mathrm{J}$ Impot Res 2002; 14:226-244.

13. Rosen RC, Cappelleri JC, Smith MD, Lipsky J, Peña BM. Development and evaluation of an abridged, 5-item version of the International Index of Erectile Function (IIEF-5) as a diagnostic tool for erectile dysfunction. Int $\mathbf{J}$ Impot Res 1999; 11:319-326.

14. Kim N, Thrash B, Menter A. Comorbidities in psoriasis patients. Semin Cutan Med Surg 2010; 29:10-15.

15. Abd El-Salam OH, El-Esawy FM, Fayed NM, Ismael YM. Assessment of some systemic affection in psoriatic patients. J Derm Venereol 2011; 31:18-25.

16. Ji S, Zang Z, Ma H, Gu M, Han Y, Wang L. Erectile dysfunction in patients with plaque psoriasis: the relation of depression and cardiovascular factors. Int J Impot Res 2016; 28:96-100.

17. Pietrzak A, Michalak-Stoma A, Chodorowska G, Szepietowski JC. Lipid disturbances in psoriasis: an update. Mediators Inflamm 2010; 2010:535612.

18. Mufaddel A, Abdelgani AE. Psychiatric comorbidity in patients with psoriasis, vitiligo, acne, eczema and group of patients with miscellaneous dermatological diagnoses. Open J Psychiatry 2014; 4:168-175.

19. Murthy AS, Leslie K. Autoinflammatory skin disease: a review of concepts and applications to general dermatology. Dermatology 2016; 232:534-540.

20. Molina-Leyva A, Jimenez-Moleon JJ, Naranjo-Sintes R, Ruiz-Carrascosa JC. Sexual dysfunction in psoriasis: a systematic review. J Eur Acad Dermatol Venereol 2015; 29:649-655. 
21. El-Rachkidy RG, Young HS, Griffiths CE, Camp RD. Humoral autoimmune responses to the squamous cell carcinoma antigen protein family in psoriasis. J Invest Dermatol 2008; 128:2219-2224.
22. Mose M, Kang Z, Raaby L, Iversen L, Johansen C. Preferential inhibition of interleukin (IL)17C, IL-1 beta and p38 mitogen-activated protein kinase in psoriatic skin by antitumour necrosis factor-alpha therapy. Br J Dermatol 2008; 159:1397-1398. 